www.nature.com/ejhg

\title{
Multiple origins of the mtDNA 7472insC mutation associated with hearing loss and neurological dysfunction
}

\author{
Tim P Hutchin ${ }^{* 1}$, Nuria C Navarro-Coy ${ }^{1}$, Guy Van Camp ${ }^{3}$, Valeria Tiranti ${ }^{4}$, \\ Massimo Zeviani ${ }^{4}$, Markus Schuelke ${ }^{5}$, Michaela Jaksch ${ }^{6}$, Valerie Newton ${ }^{7}$ and \\ Robert F Mueller ${ }^{2}$
}

\begin{abstract}
${ }^{1}$ Molecular Medicine Unit, University of Leeds, Leeds, UK; ${ }^{2}$ Department of Clinical Genetics, St James's University Hospital, Leeds, UK; ${ }^{3}$ Department of Medical Genetics, University of Antwerp, Belgium; ${ }^{4}$ Division of Biochemistry and Genetics, National Neurological Institute 'C. Besta', Milan, Italy; ${ }^{5}$ Department of Neuropediatrics Charite, Virchow University Hospital, Berlin, Germany; ${ }^{6}$ Institute of Clinical Chemistry, Molecular Diagnostic and Mitochondrial Genetics, Academic Hospital Schwabing, Munich, Germany; ${ }^{7}$ Centre for Audiology, Education of the Deaf and Speech Pathology, University of Manchester, Manchester, UK
\end{abstract}

Several mtDNA mutations have been reported in families with both syndromic and non-syndromic hearing loss. One such mutation is the heteroplasmic 7472insC in the tRNA ${ }^{\text {Ser(UCN) }}$ gene which has been found in six families, all from Western Europe. However, it was not clear if this distribution was due to a common founder effect or chance sampling of several unrelated families, the 7472insC mutation having occurred multiple times. Haplotype analysis of all six families supports the latter notion. This confirms the pathogenicity of the 7472ins $\mathrm{C}$ mutation and suggests it may exist in other populations where it may prove to be a small but significant cause of hearing loss, particularly when neurological symptoms are also present. European Journal of Human Genetics (2001) 9, $385-387$.

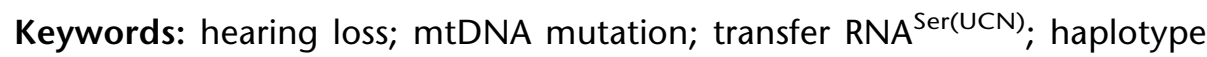

\begin{abstract}
Mutations of the mitochondrial DNA (mtDNA) have been associated with a variety of human diseases. Due to selective pressures the more severe of these mutations exist in the heteroplasmic state on different mtDNA backgrounds whereas mild mutations tend to be homoplasmic and are often restricted to a particular mtDNA background. Phylogenetic analysis of mtDNA sequences allows one to determine if a particular mutation has occurred multiple times on different genetic backgrounds or if all affected individuals
\end{abstract}

*Correspondence: Tim P Hutchin, Molecular Medicine Unit, Clinical Sciences Building, St James's University Hospital, Beckett Street, Leeds LS9 7TF, UK.

Tel: +44 113206 5696; Fax: +44 113244 4475;

E-mail: t.p.hutchin@leeds.ac.uk

Received 17 November 2000; revised 23 January 2001; accepted 31 January 2001 are derived from a common ancestor, ie there is a founder effect. The former strengthens the case for the mutation being pathogenic and allows one to see if any variation in disease between affected individuals can be attributed to variation elsewhere in the mtDNA or nuclear DNA. Furthermore, the finding of the same mutation in unrelated individuals suggests the mutation is equally likely to exist on mtDNA from any population.

Recent analysis of the A1555G mutation, one of the most common genetic causes of hearing loss identified, showed the mutation has occurred multiple times in both Asian and European populations. ${ }^{1,2}$ Among several other mtDNA mutations shown to cause hearing loss is a heteroplasmic 1 base pair insertion at $n p 7472$ in the $\mathrm{tRNA}^{\mathrm{Ser}(\mathrm{UCN})}$ gene. In some individuals where levels of the mutation are greater than 95\% neurological features such as ataxia and myoclonus also occur. ${ }^{3,4}$ 
Table 1 Haplogroup classification of the 7472insC families. The presence $(+)$ or absence ( - ) of each specific restriction enzyme site is shown for each family with the 7472insC mutation. Each family was then assigned to a particular mtDNA haplogroup. Families are from Italy (It), the Netherlands (NI), Germany (G1-G3) and the UK (UK)

\begin{tabular}{|c|c|c|c|c|c|c|c|c|c|c|c|}
\hline \multicolumn{12}{|c|}{ Sites\# } \\
\hline Individual & $\begin{array}{c}-1715 \\
\text { Ddel }\end{array}$ & $\begin{array}{c}-4529 \\
\text { HaellI }\end{array}$ & $\begin{array}{c}-4577 \\
\text { Nlalll }\end{array}$ & $\begin{array}{c}-7025 \\
\text { Alul }\end{array}$ & $\begin{array}{c}-8994 \\
\text { Haelll }\end{array}$ & $\begin{array}{c}-9052 \\
\text { Haell }\end{array}$ & $\begin{array}{c}+10397 \\
\text { Alul }\end{array}$ & $\begin{array}{c}+12308 \\
\text { Hinfl }\end{array}$ & $\begin{array}{c}+13366 \\
\text { BamHI }\end{array}$ & $\begin{array}{c}-13704 \\
\text { BstNI }\end{array}$ & Haplogroup \\
\hline UK & - & - & - & + & - & - & - & - & - & - & $\mathrm{H}$ \\
\hline $\mathrm{NI}$ & - & - & - & - & - & + & - & + & - & - & K \\
\hline It & - & - & - & + & - & - & - & - & - & - & $\mathrm{H}$ \\
\hline G1 & - & - & - & + & - & - & - & - & - & - & $\mathrm{H}$ \\
\hline G2 & - & - & + & - & - & - & - & - & - & - & V \\
\hline G3 & - & - & - & + & - & - & - & - & - & - & $\mathrm{H}$ \\
\hline
\end{tabular}

To date the 7472insC mutation has been found in six families, all from Western Europe, ${ }^{3-6}$ raising the possibility that the presence of this mutation in Europe is primarily due to a founder effect. Alternatively, the finding may be due to chance sampling, the 7472insC mutation having occurred multiple times on different mtDNA backgrounds. If the latter is the case then the 7472insC mutation may be present in other populations and may prove to be an important cause of hearing loss and neurological dysfunction.

\section{Materials and methods \\ Patients}

DNA samples from one individual of each of the six affected families were as follows (see Table 1):

It: The first family described with the 7472insC mutation originating from Italy. ${ }^{3}$ Affected individuals suffer sensorineural hearing loss often with ataxia and myoclonus when the mutation is present at greater than $95 \%$.

G1-3: These three families from Germany have previously been described ${ }^{4,5}$ corresponding to families A, B and C. All index patients had sensorineural hearing loss and progressive myoclonic epilepsy.

$\mathrm{Nl}$ Affected individuals of this family from the Netherlands suffer from sensorineural hearing loss, only one individual showed neurological symptoms including ataxia and dysarthria. ${ }^{6}$

UK: This individual with non-syndromic sensorineural hearing loss originates from the UK. Although other family members had the 7472insC mutation their hearing was normal (to be described elsewhere).

\section{mtDNA haplogroup analysis}

Previous studies have shown that the majority of Caucasians can be split into 10 different mtDNA haplogroups on the basis of 10 polymorphic sites. ${ }^{7}$ Each site was detected by PCR amplification of the relevant region and digestion with the appropriate restriction enzyme as shown in Table 1 . The
Table 2 Sequencing of non-coding D-loop region of the 7472insC families. Base changes in the D-loop are given for each individual relative to the Cambridge reference sequence (Ref). The polymorphic T16519C site was determined by digestion with Haell

\begin{tabular}{llllllllllll}
\hline & 1 & 1 & 1 & 1 & 1 & 1 & 1 & 1 & 1 & 1 & 1 \\
& 6 & 6 & 6 & 6 & 6 & 6 & 6 & 6 & 6 & 6 & 6 \\
& 0 & 1 & 1 & 2 & 2 & 2 & 3 & 3 & 3 & 4 & 5 \\
& 9 & 1 & 8 & 2 & 7 & 9 & 1 & 5 & 6 & 4 & 1 \\
& 3 & 4 & 9 & 4 & 8 & 8 & 1 & 6 & 3 & 7 & 9 \\
$\operatorname{Ref}$ & $T$ & $C$ & $T$ & $T$ & $C$ & $T$ & $T$ & $T$ & $T$ & $C$ & $T$ \\
UK &. &. &. &. &. &. &. &. &. &. &. \\
$I t$ &. &. &. &. & $T$ &. & $\mathrm{C}$ &. &. &. & $C$ \\
$\mathrm{G} 1$ &. &. & $\mathrm{C}$ &. &. &. &. & $\mathrm{C}$ & $\mathrm{C}$ &. & $\mathrm{C}$ \\
$\mathrm{G} 3$ &. &. &. &. &. &. &. &. &. &. & $\mathrm{C}$ \\
$\mathrm{NI}$ & $\mathrm{C}$ &. &. & $\mathrm{C}$ &. &. & $\mathrm{C}$ &. &. & $T$ & $\mathrm{C}$ \\
$\mathrm{G} 2$ &. &. &. &. &. & $\mathrm{C}$ & $\mathrm{C}$ &. &. &. &. \\
\hline
\end{tabular}

polymorphism at position 12308 was detected using a mismatch primer to create a Hinfl site.

\section{mtDNA sequence analysis}

The non-coding D-loop region was amplified using primers mt1592 (5' actaatacaccagtcttgtaaacc) and mt16560 (5' gacctgtgatccatcgtgatg) and sequenced using ABI big dye terminator kit with internal forward primer $\mathrm{mt} 1598$ gaaaagtctttaactccacc) and reverse primer mt1649 (5' cctgaagtaggaaccagatg). The presence of the polymorphic 16517 HaeIII site was also screened for in this PCR product.

\section{Results}

As shown in Table 1, all six affected individuals could be classified into one of the Caucasian mtDNA haplogroups as previously defined. ${ }^{7}$ The individual from The Netherlands (Nl) harboured a loss of the HaeIII site at position 9052 and the 12308G polymorphism, both of which define haplogroup K. The second family from Germany (G2) lacked the 4577 NlaIII site, unique to haplogroup V. All other 
samples lacked the 7025 AluI site, characteristic of haplogroup $\mathrm{H}$. The presence of four individuals from haplogroup $\mathrm{H}$ is not unexpected given that this is the most common ( 40\%) European haplogroup. ${ }^{7}$

D-loop sequence analysis of the four haplogroup $\mathrm{H}$ families showed they were not closely related, all differing from one another by at least 2 base changes (Table 2). In addition, patient $\mathrm{Nl}$ harboured the T16224C polymorphism which defines haplogroup $\mathrm{K}$, in agreement with the RFLP analysis.

\section{Discussion}

Our analysis of mtDNA from the six European families with the 7472insC mutation shows that it is randomly distributed among different haplogroups in the Caucasian population. The simplest explanation is that the mutation has occurred multiple times in the European population, each time resulting in hearing loss and in many cases also neurological features. Taken together this analysis implies the 7472insC mutation has most probably occurred up to six times in the Western European population.

The presence of the same mutation on separate mtDNA backgrounds confirms the notion that the 7472insC mutation alone is sufficient to cause hearing loss and that when present at very high levels can also lead to neurological dysfunction. It has been shown that the mutation causes a $65 \%$ reduction in levels of $\mathrm{tRNA}^{\mathrm{Ser}(\mathrm{UCN})}$ although it results in only a small decrease in mitochondrial respiratory enzyme activities. ${ }^{3,8}$ Why this defect appears most pronounced in the inner ear is not yet known.

mtDNA mutations are now well recognised as causes of hearing loss. However, little data is available on the frequency of mutations such as 7472insC, A1555G and A3243G in individuals with hearing loss. Although the 7472 insC mutation was not present in 117 individuals with familial hearing loss from Finland the A1555G and A3243G mutations were present in $2.5 \%$ and $4.3 \%$ of individuals respectively. ${ }^{9}$ The A1555G mutation was present in $5.4 \%$ of Japanese individuals with hearing loss ${ }^{10}$ and in Spain this figure was $27.7 \%$ in familial, progressive hearing loss. ${ }^{11}$ Such differences suggest population differences do exist, although selection criteria of the individuals may also account for some of these differences.

Despite the absence of the 7472insC mutation in the Finnish study and 249 other cases of childhood onset hearing loss from the UK in our study, screening for the 7472insC mutation is, nevertheless, worth considering in any case of progressive sensorineural hearing loss, particularly if neurological symptoms such as ataxia, dysarthria and myoclonus are also present. Our findings that the 7472 insC mutation has occurred multiple times also means that it is perhaps not unreasonable to expect the mutation to be present in other populations. Further studies are required to determine the frequency of the 7472insC mutation in other populations and shed further light into the pathogenic mechanism by which mitochondrial dysfunction leads to hearing loss and neurological dysfunction.

\section{Acknowledgments}

Work in the authors' laboratories is supported by the National Lottery Charities Board through Defeating Deafness, The Royal National Institute for Deaf People, the West Riding Medical Research Trust, the Medical Research Council, European Community Framework $V$ and the Wellcome Trust.

\section{References}

1 Hutchin TP, Cortopassi GA: Multiple origins of a mitochondrial mutation conferring deafness. Genetics 1997; 145: 771-776.

2 Torroni A, Cruciani F, Rengo C et al: The A1555G mutation in the $12 \mathrm{~S}$ rRNA gene of human mtDNA: recurrent origins and founder events in families affected by sensorineural deafness. Am J Hum Genet 1999; 65: 1349-1358.

3 Tiranti V, Chariot P, Carella F et al: Maternally inherited hearing loss, ataxia and myoclonus associated with a novel point mutation in mitochondrial tRNASer(UCN) gene. Hum Mol Genet 1995; 4: $1421-1427$

4 Jaksch M, Klopstock T, Kurlemann G et al: Progressive myoclonus epilepsy and mitochondrial myopathy associated with mutations in the tRNA(Ser(UCN)) gene. Ann Neurol 1998; 44: $635-640$

5 Schuelke M, Bakker M, Stoltenburg G, Sperner J, von Moers A: Epilepsia partialis continua associated with a homoplasmic mitochondrial tRNA(Ser(UCN)) mutation. Ann Neurol 1998; 44: $700-704$.

6 Verhoeven K, Ensink RJH, Tiranti V et al: Hearing impairment and neurological dysfunction associated with a mutation in the mitochondrial tRNASer(UCN) gene. Eur J Hum Genet 1999; 7: $45-51$.

7 Torroni A, Huoponen K, Francalacci P et al: Classification of European mtDNAs from an analysis of three European populations. Genetics 1996; 144: $1835-1850$.

8 Toompuu M, Tiranti V, Zeviani M, Jacobs HT: Molecular phenotype of the np 7472 deafness-associated mitochondrial mutation in osteosarcoma cell cybrids. Hum Mol Genet 1999; 8: $2275-2283$

9 Lehtonen MS, Uimonen S, Hassinen IE, Majamaa K: Frequency of mitochondrial DNA point mutations among patients with familial sensorineural hearing impairment. Eur J Hum Genet 2000; 8: 315-318.

10 Usami S, Abe S, Akita J et al: Prevalence of mitochondrial gene mutations among hearing impaired patients. J Med Genet 2000; 37: $38-40$.

11 Estivill X, Govea N, Barcelo A et al: Familial progressive sensorineural deafness is mainly due to the mitochondrial DNA A1555G mutation and is enhanced by treatment with aminoglycosides. Am J Hum Genet 1998; 62: 27-35. 